Cipango Cahiers d'études japonaises

$20 \mid 2013$

Nouveaux regards sur les arts de la scène japonais I

\title{
Le rakugo et Henry Black
}

Comment un Britannique conta la modernité à Meiji

Rakugo and Henry Black: How a Briton used the Art of Storytelling On Stage in Meiji Era Japan

\section{Ian McArthur}

\section{(2) OpenEdition Journals}

\section{Édition électronique}

URL : https://journals.openedition.org/cipango/1990

DOI : 10.4000/cipango.1990

ISSN : 2260-7706

\section{Éditeur}

INALCO

Édition imprimée

Date de publication : 30 octobre 2013

ISSN : 1164-5857

\section{Référence électronique}

Ian McArthur, «Le rakugo et Henry Black », Cipango [En ligne], 20 | 2013, mis en ligne le 16 avril 2015, consulté le 30 juin 2021. URL : http://journals.openedition.org/cipango/1990 ; DOI : https://doi.org/ 10.4000/cipango. 1990

Ce document a été généré automatiquement le 30 juin 2021.

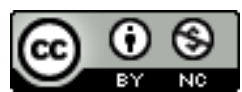

Cipango est mis à disposition selon les termes de la Licence Creative Commons Attribution - Pas d'Utilisation Commerciale 4.0 International. 


\title{
Le rakugo et Henry Black
}

\author{
Comment un Britannique conta la modernité à Meiji \\ Rakugo and Henry Black: How a Briton used the Art of Storytelling On Stage in \\ Meiji Era Japan
}

Ian McArthur

1 Cet article est l'adaptation d'une présentation intitulée « Le Rakugo, à travers la vie du conteur Henry Kairakutei Black» donnée au Centre culturel Bertin Poirée à Paris le 24 octobre 2011, et suivie d'une interprétation de rakugo en hommage à Henry Black par le conteur.

2 Le rakugo 落語 est l'art de conter des histoires, la plupart du temps brèves et comiques ${ }^{1}$. Le conteur, assis sur un coussin que l'on appelle zabuton, reproduit les voix et actions des personnages de l'histoire. Il utilise peu d'accessoires. Seulement un chiffon appelé tenugui et un éventail. Le tenugui peut devenir portefeuille, couvre-chef, ou mouchoir, l'éventail se transformer en pipe, épée, baguettes, ou rame de bateau.

3 De nombreux récits du répertoire classique du rakugo puisent leur origine dans les paraboles bouddhiques ou dans les collections d'histoires telles que le Nihon ryōiki ${ }^{2}$. Celles-ci contenaient des thèmes que l'on retrouve dans le rakugo, tels que le renard déguisé ou le crâne humain qui revient à la vie. Parmi les autres grandes influences, on citera les prédications des moines bouddhistes et les histoires guerrières racontées par les conteurs $\mathrm{du} \mathrm{xvI}$ siècle. Ces formes de narration furent adoptées par des laïcs et évoluèrent pour devenir un divertissement complètement séculaire. Lieux de divertissement où se tenaient avant tout des performances musicales et autres actes de jonglage, les théâtres, qui connaissent une popularité affirmée à la fin du XVIII ${ }^{\mathrm{e}}$ siècle, accueillent les conteurs et leurs apprentis à partir de 1790. L'avènement de personnes interprétant des histoires pour un auditoire de manière régulière marque l'émergence d'une forme d'art populaire destinée au grand public. Cependant, ce phénomène ne prit de l'ampleur qu'à partir des années $1840^{3}$.

4 Les histoires de rakugo ont une structure qui inclut un prologue, le corps du récit, et une chute que l'on appelle ochi (落, qui se lit également raku, d'où le terme de rakugo). L'ochi doit fournir une sorte de catharsis ou de relâchement pour les auditeurs. Il doit 
faire rire, même si la partie principale de l'histoire a pu émouvoir. Un certain nombre d'artifices sont employés pour atteindre cet objectif, parmi lesquels on note l'utilisation fréquente de la pause ou moment de silence, appelé $m a$ 間, en des points cruciaux de la narration.

Les premières "lignées" de conteurs (c'est-à-dire les groupes de conteurs qui s'entraînaient sous l'égide d'un patron et qui se représentaient sous le même nom de scène lorsqu'ils devenaient professionnels) semblent être apparues aux alentours des années 1770. Elles se sont multipliées au milieu des années 1880. Pour devenir un conteur professionnel (rakugoka 落語家), de longues années de formation sont nécessaires. Une cérémonie particulière pouvait marquer l'obtention du statut de maître-conteur (shin.uchi 真打) ${ }^{4}$.

6 Le répertoire établi à la fin d'Edo évoque avant tout les gens ordinaires, le petit peuple d'Edo ou d'Ōsaka (on peut d'ailleurs distinguer deux traditions différentes, celle d'Edo, et celle du Kamigata, la région d'Ōsaka). Il inclut également des histoires mettant en scène les protagonistes des quartiers de plaisir, des récits dans lesquels le personnage central part en voyage (parfois au paradis ou en enfer) et rencontre toutes sortes d'animaux étranges ou de situations amusantes, des histoires de voleurs ineptes, ainsi que des contes sur des samouraïs stupides ou des prêtres bouddhistes. Les personnages typiques sont le seigneur (tonosama 殿様), le maître de maison (danna 旦那), le jeune maître de maison (wakadanna 若旦那), l'homme de compagnie (taikomochi 堼間), la courtisane (oiran 花魁). Une bonne partie du répertoire du rakugo se moque des figures de l'autorité et fait preuve d'un humour qu'il faut bien avouer assez farce. Les titres évoquent des thèmes susceptibles d'intéresser et de faire rire: le Gardien paresseux des bains publics, l'Apprivoisement du fantôme, la Supercherie du superstitieux de la maison de thé, le Chien illettré, le Long Sous-vêtement, pour n'en citer que quelques-uns ${ }^{5}$. Ce premier corpus est complété par les rakugoka de l'ère Meiji, et en particulier par le mentor du personnage dont il est question dans cet article, San.yūtei Enchōo ${ }^{6}$.

7 Les salles de rakugo sont appelés yose. De nos jours, à Tōkyō, il n'y a plus que cinq grands établissements de ce genre, le Suzumoto engeijo 鈴元演芸場 à Ueno, le Suehirotei 末廣亭 à Shinjuku, l'Ikebukuro engeijō 池袋演芸場, l'Asakusa engei hōru 浅 草演芸ホール et la salle nationale Kokuritsu engeijō 国立演芸場 à Nagata-chō.

8 L'âge d'or du rakugo fut l'ère Meiji. Cela peut étonner : alors que celle-ci est associée à une occidentalisation et une modernisation effectuées à marche forcée, comment un art du conte traditionnel a-t-il donc pu survivre et prospérer? Pourtant, au plus haut de sa popularité, en particulier durant les années 1880 à 1890, le rakugo jouit bien d'un statut sans précédent en tant que forme de divertissement. Tōkyō accueille alors jusqu'à deux cent trente yose et le rakugo est un des arts scéniques les plus répandus et accessibles à tous ${ }^{7}$.

9 On peut évoquer plusieurs raisons pour expliquer cette popularité, la cause principale étant certainement que le rakugo constituait une forme de divertissement peu onéreuse et accessible aux gens ordinaires. Dans les théâtres yose, dont l'entrée était bien moins chère que pour le kabuki, ceux-ci pouvaient apprécier, outre les conteurs qui constituaient le divertissement principal et étaient de vraies célébrités, des acrobates, des musiciens... En 1905, l'Écossais Francis McCullagh de visite au Japon fut si impressionné par l'importance de cette forme de divertissement qu'il nota dans son journal intime que les conteurs de rakugo étaient « aussi nombreux que populaires » et 
que les yose étaient aussi répandus dans les villes et villages japonais que les pubs en Irlande ${ }^{8}$ !

10 Une seconde raison est la vertu que l'on reconnaissait au yose, et qui n'est pas sans rapport avec le caractère édifiant qu'il avait endossé aux époques précédentes. À partir de l'ère Meiji, le rakugo fut généralement reconnu par le gouvernement comme ayant une fonction éducative. Les apprentis venant de la campagne pour travailler à Tōkyō étaient par exemple emmenés au yose pour y apprendre les usages du monde ${ }^{9}$. Au-delà $\mathrm{du}$ divertissement, les conteurs remplissaient donc une fonction sociale très importante. Art pour le peuple ordinaire, le rakugo connut une très large popularité durant ces années. Mais il sut également séduire bon nombre d'intellectuels de Meiji, comme Fukuzawa Yukichi, qui assistait régulièrement à des représentations, ou Natsume Sōseki, qui reconnaissait l'influence du rakugo sur son œuvre ${ }^{10}$.

\section{Le conteur aux cheveux clairs}

11 À cette époque, certains conteurs clairvoyants et innovants adaptèrent des histoires occidentales à leur art. Le guide de cette nouvelle tendance fut San.yūtei Enchō 三遊亭 円朝 (1839-1900). Avec l'aide de certaines de ses connaissances qui comprenaient l'anglais et le français, Enchō traduisit et adapta par exemple la nouvelle de Maupassant Un Parricide avec la pièce Meijin Chōji 名人長二 (le Maître ébéniste Chōji) ${ }^{11}$, la Tosca de Victorien Sardou ou le roman Hard Cash de Charles Reade ${ }^{12}$.

12 Mais il y eut un autre rakugoka japonais dont on pourrait dire qu'il surpassa Enchō dans ses adaptations d'histoires occidentales. Il fut en tout cas l'un des conteurs réformistes japonais les plus populaires de tous les temps. Son nom de scène était Kairakutei Burakku 快楽亭ブラック, né Henry Black, à Adelaïde, petite ville britannique du sud de l'Australie en 1858, et citoyen britannique au moment de sa naissance.

La famille Black déménagea à Yokohama en 1865. Son père, John Black, devint en effet l'éditeur en chef du journal publié en anglais, le Japan Herald. Pendant sa carrière, Henry Black créa de très nombreuses histoires originales, mais interpréta également des rôles de kabuki, et aida à la production des premiers vinyles fabriqués au Japon. Il pratiqua l'hypnose et eut même à un certain moment son propre groupe de musique de style occidental. Il devint aussi citoyen japonais, en 1893. Ce fut, en bref, un Japonais d'origine britannique né en Australie extrêmement talentueux.

Le parcours de Black ne cesse de surprendre. Comment quelqu'un dont l'origine étrangère est si flagrante a-t-il pu jouer dans les théâtres du Japon dans les années 1880 ? La réponse tient au fait qu'il vécut au Japon à une période de bouleversements sociaux sans précédent, en un temps où la population était très ouverte à toutes sortes de nouveautés.

Entre mai et novembre 1879, à la requête de plusieurs sociétés cherchant à promouvoir la démocratie, John et Henry Black prirent la parole dans un certain nombre de meetings publics à Tōkyō et dans ses environs sur des sujets concernant les modes de gouvernement et le système législatif ${ }^{13}$. Henry Black avait alors à peine plus de vingt ans. Leur première intervention fut un discours à une réunion du Kun.yūsha 薰誘舎 à Yūrakuchō le premier juin, au cours de laquelle ils s'y sont exprimés sur la question alors brûlante des droits du peuple (minkenron 民権論). Leurs sujets ultérieurs inclurent les avantages et désavantages du système pénitentiaire, Napoléon ${ }^{14}$, la fermeture du 
quartier de plaisir de Yoshiwara, les effets négatifs de l'ouverture du Japon, les procédures criminelles, la prévention contre le choléra, l'importance d'un jury au tribunal, les formes de gouvernement (seitairon 政体論), le jugement sur la base de preuves (shōko saiban no setsu 証拠裁判の説), et les relations entre le peuple et le gouvernement (jinmin to seifu no kankei 人民と政府の関倸). Ces sujets constituèrent des thèmes clés qu'Henry Black inclura plus tard dans ses histoires.

La capacité de Black à parler japonais attira l'attention de conteurs professionnels qui l'invitèrent à venir parler dans des yose. L'un de ceux-ci fut Shōrin Hakuen 松林伯円, un éminent adepte du style didactique ( $k \bar{d} d a n$ 講談), qui lui enseigna l'art de conter. L'une des premières interventions d'Henry Black eut lieu en décembre 1878 au théâtre Tomitake-tei 富竹亭, dans la fameuse rue des théâtres Bashamichi 馬車道 à Yokohama ${ }^{15}$. À cette occasion, il raconta l'histoire de Jeanne d'Arc et celle de l'exil du promis au trône d'Écosse, Charles Edward Stuart. La mythologie entourant Charles Edward Stuart et Jeanne d'Arc, deux héros "nationaux », n'était sans doute pas sans intérêt pour le public japonais, la quête d'une identité nationale faisait déjà partie intégrante de la recherche japonaise de modernité. Ces histoires servaient aussi de mise en garde contre la menace coloniale britannique, dont les prouesses militaires semblaient en partie explicables par une certaine disposition philosophique. Le choix de Jeanne d'Arc et de Charles Edward Stuart suggère donc qu'Henry Black était particulièrement sensible à l'idéalisme nationaliste des États-nations européens du XIX ${ }^{\mathrm{e}}$ siècle et qu'il avait perçu l'attrait que cet idéalisme possédait pour de nombreux Japonais. Il démontre encore l'adresse avec laquelle il sut entendre et répondre à une attente du public.

On voit déjà dans cette représentation les qualités que Black démontrera tout au long de sa carrière de conteur : une aisance et une assurance à s'exprimer dans la langue japonaise en public, une connaissance des affaires nationales et internationales ainsi que des cultures occidentales et japonaises, et un talent pour choisir des thèmes qui avaient de l'intérêt pour le public japonais. S'ajoute à cela sa spécificité en tant que narrateur d'ascendance européenne.

Henry Black avait vingt-sept ans quand en 1886 il cessa d'enseigner l'anglais à plein temps et donna la première représentation théâtrale de son feuilleton la Rosée du cimetière $^{16}$, qu'il avait adaptée du roman Flower and Weed de l'auteur britannique Mary Braddon ${ }^{17}$.

Black s'associa officiellement avec la compagnie San.yū en septembre 1890, à l'âge de trente-et-un ans. En mars 1891, il prit le nom de scène Kairakutei Burakku 快楽亭ブ ラック après avoir atteint le statut de shin.uchi dans la compagnie ${ }^{18}$. Henry expliqua plus tard que les membres de la troupe avaient souhaité qu'il les rejoigne en pensant que le caractère insolite d'un rakugoka occidental pouvait assurer un certain succès ${ }^{19}$. Il ne fait en effet aucun doute que les membres les plus gradés de la compagnie virent en cet étranger qu'était Black une possibilité d'innover le répertoire du rakugo. 
Henry Kairakutei Black, assis sur son estrade

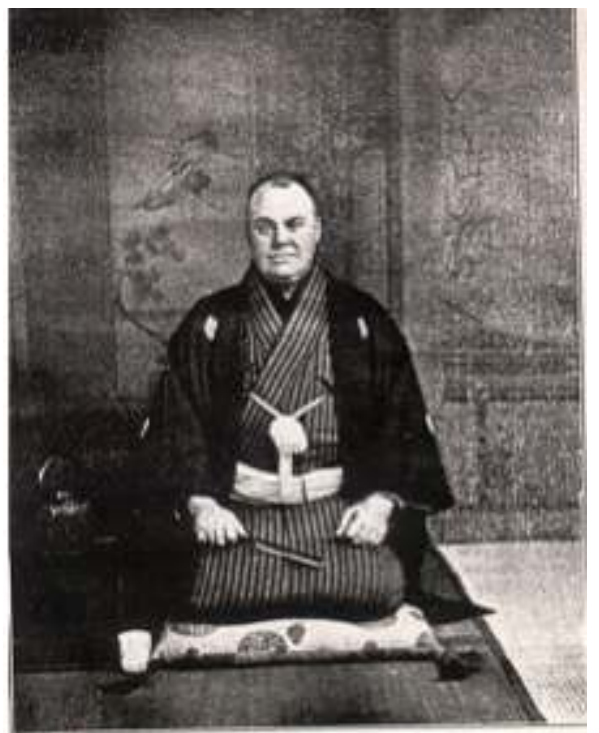

Henry Kairakutei Black, assis sur son estrade ; "The Story Teller in Japan", par Francis McCullough, in East of Asia Magazine, no. 1, North China Herald Office, Shanghai, 1902, p. 218. objet de curiosité. Lors de sa prise de nom de scène, l'édition du 24 mars 1891 du Yamato shinbun titra ainsi : «L'Anglais Black à la couleur des cheveux étrange promu au rang de rakugoka $»^{20}$. Par certains aspects, Black jouait de cet exotisme : bien que la plupart des rakugoka étaient sur scène assis sur un zabuton avec leurs jambes repliées sous euxmêmes, des illustrations de Black durant cette période indiquent qu'il pouvait conter assis sur une chaise, derrière une petite table sur laquelle se tenaient un verre et une carafe d'eau. On perçoit ici l'influence du kōdan, où le conteur est assis devant une table basse qu'il frappe de temps en temps de son éventail, mais également de la mode des discours de type occidental à l'ère Meiji.

21 Le succès vint rapidement : comme tous les rakugoka extrêmement populaires, Black pouvaient donner des représentations dans plusieurs yose de suite durant la même journée. Un conducteur de pousse-pousse l'attendait à l'extérieur du théâtre pour l'emmener à son prochain spectacle le plus vite possible. le Français Jules Adam, premier secrétaire de la délégation française. Celui-ci était tout particulièrement intéressé par les rakugoka dont il disait qu'ils étaient «une classe d'artistes curieux et remarquables", rapportant que les yose tenaient «une place prépondérante dans l'existence des Japonais ». Dans le livre qu'il écrivit en français au sujet du rakugo, et dont les citations précédentes sont tirées ${ }^{21}$, Adam mentionne effectivement Henry Black, pour lequel il éprouvait une véritable fascination.

J'avais été hanté depuis longtemps par le désir secret de faire connaissance avec ce phénomène, ce dilettante ou ce marginal - je ne sais comment le décrire - à l'égard duquel je nourrissais une admiration étrange mélangée à de la sympathie et de la curiosité. Je voulais lui poser des questions, l'étudier, tourner une à une les pages de sa vie, comme l'on ferait avec un livre ou autre document.

Un jour, Adam séjourna dans une auberge, dans la source thermale d'Ikaho au nord de Tōkyō, où Black était passé la veille. L'aubergiste étant incapable de lui dire où Black 
s'en était allé, Adam fut très déçu. Mais quelques mois plus tard, à Kōbe, Adam remarqua que Black donnait une représentation dans un théâtre de la ville.

J'entrai et eus la chance de l'entendre pour la première fois. J'étais époustouflé. La salle tremblait encore des applaudissements du public quand soudain il quitta la scène. Je me hâtai au-dehors, pressé de me présenter à lui et de le féliciter, mais il était déjà parti. Sautant dans une jinrikisha, je me rendis alors à son hôtel, ayant réussi à me procurer son adresse. Hélas ! L'oiseau s'était déjà envolé. Il avait attrapé le train de minuit pour se rendre à un rendez-vous dans une importante ville du $\operatorname{sud}^{22}$.

Le succès que connut Black l'envoyait ainsi en tournées dans tout le Japon, mais bientôt, et dès le début des années 1880 , il connut des difficultés pour voyager librement à travers le pays. Le gouvernement avait en effet placé des restrictions sur les déplacements des étrangers au dehors des villes de Tōkyō, Ōsaka et Kōbe. Sa solution fut de se marier pour se faire adopter par la famille de son épouse japonaise ${ }^{23}$. Henry Black contracta donc en 1893, à l'âge de trente-trois ans, un mariage de convenance avec Ishii Aka 石井アカ, dont l'édition du Chūō shinbun 中央新聞 du 24 mai 1893 explique qu'elle est fille d'Ishii Mine, a dix-huit ans, et travaille dans une confiserie $^{24}$. Il est ainsi adopté par la famille Ishii, dont il acquiert le nom pour devenir officiellement Ishii Burakku, citoyen japonais ${ }^{25}$. Black étant homosexuel, son mariage ne fut pour lui qu'un moyen pour obtenir la citoyenneté japonaise et la liberté de mouvement au Japon. Il continua à vivre comme précédemment avec son compagnon Takamatsu Motokichi. Cette relation est d'ailleurs attestée par le rapport officiel de l'examen du statut matrimonial de Black, qui précise qu'il est "conteur en langue japonaise» (nihongo o motte kōdan o gyō to suru 日本語を以って講談を業とする), signale qu'il préfère habituellement les hommes (tsune ni nanshoku o konomu 常に男色 を好む), et qu'« il est très attaché au roturier (heimin) originaire de Tōkyō, Takamatsu Motosuke, avec lequel il semble pratiquement entretenir une relation de couple ${ }^{26}$.

Jules Adam, Japanese Storytellers - From the French of Jules Adam

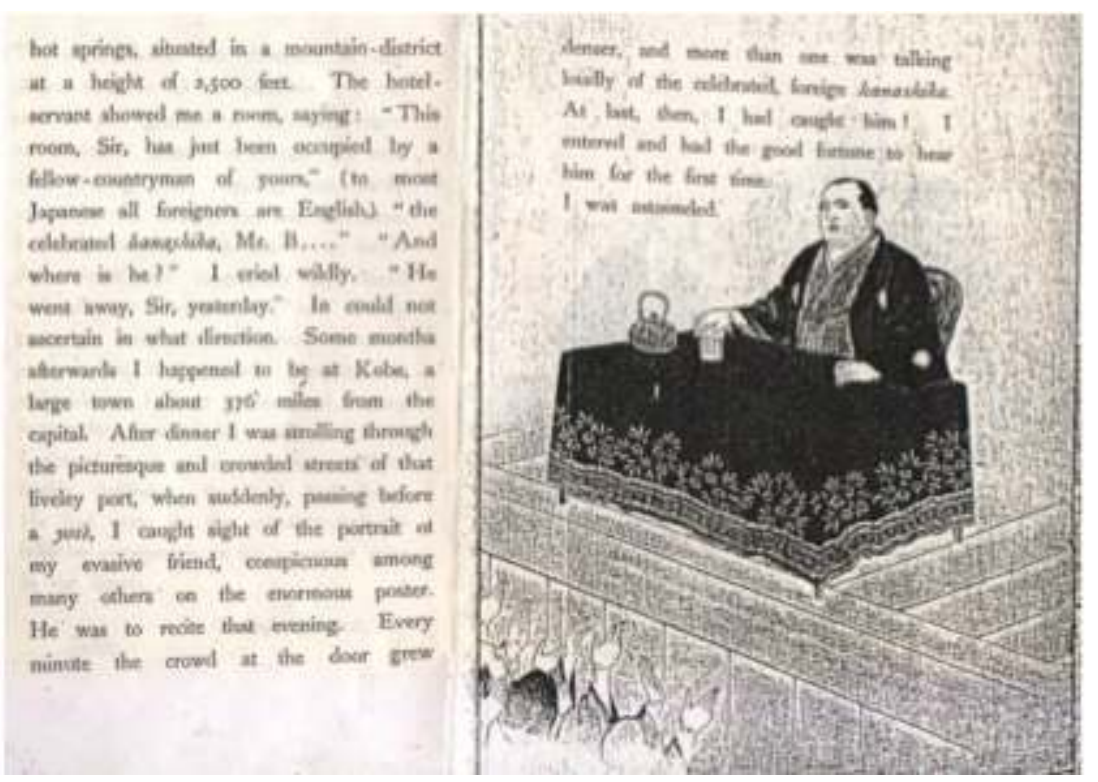

Jules Adam, Japanese Storytellers - From the French of Jules Adam, traduit par Osman Edwards, T. Hasegawa, Tōkyō, 1900, (édition française 1899), p. 20. 


\section{Adapter des romans occidentaux pour parler de la société japonaise}

Black devint donc un éminent rakugoka, connu pour adapter un grand nombre d'histoires d'origine étrangère. Certes, comme tous les conteurs, Black mettait en scène des aventures drolatiques dont il avait été théoriquement le témoin, ou concrètement le protagoniste. C'est ainsi qu'il racontait le souvenir d'un incident durant lequel, ayant très faim, il était entré dans un restaurant et avait commandé un grand nombre de bols de nouilles. La serveuse ne lui avait pourtant pas apporté son repas alors qu'elle avait servi plusieurs autres clients qui eux mangèrent tous à leur faim puis s'en allèrent. Finalement, Henry avait fait signe de nouveau à la serveuse et exigé de savoir pourquoi il n'avait pas été servi. Et de s'entendre dire qu'il voulait sans doute attendre ses amis avant de manger puisqu'il avait commandé autant de bols ${ }^{27}$ !

Pourtant la matière première des histoires de Black semble avoir eu une origine principalement anglaise et française. Dès 1901, il affirma ainsi «avoir traduit en japonais pas moins de quatorze romans anglais $»^{28}$. Sa capacité à traduire de l'anglais en japonais lui permit d'utiliser des genres littéraires populaires, tels que le roman policier. Comme la fin de chapitre de ces romans laisse souvent le lecteur en haleine, cela se prêtait tout particulièrement à la méthode de Black consistant à raconter des histoires en plusieurs épisodes sur plusieurs jours. J'ai déjà donné l'exemple de l'histoire de l'auteur anglais Mary Braddon qu'il utilisa à ses débuts. Une autre histoire qu'il emprunta au même auteur est sa nouvelle "Her Last Appearance ", écrite en $1876^{29}$, qu'il adapta dans ses Souvenirs du théâtre de Londres en $1891^{30}$. Il ne serait pas surprenant que d'autres histoires de Black, particulièrement celles se déroulant à Paris, trouvent leur inspiration et peut-être même leur origine dans des œuvres françaises que Braddon plagia ou adapta ${ }^{31}$. L'intrigue n'apparaît pourtant souvent que comme un prétexte pour traiter de questions qui agitaient la société de l'époque. À travers sa discussion du comportement dissolu de l'antihéros de cette histoire par exemple, Black pointait le préjudice porté à la famille et à la société par les hommes qui dilapidaient leur argent au Yoshiwara. Il abordait ainsi la question controversée de la fermeture du quartier de plaisir de Yoshiwara à Tōkyō qui était débattue dans nombre d'éditoriaux contemporains.

Le principe se vérifie dans l'une des adaptations les plus réussies que Black effectua. L'Épingle empoisonnée de l'omnibus fut écrite en 1891 à partir d'une œuvre française, le roman policier de Fortuné de Boisgobet, Le Crime de l'omnibus ${ }^{32}$. Dans la version originale, de Boisgobet met en scène deux héros: l'artiste Paul Freneuse et son compagnon Binos. Ce sont eux qui découvrent la raison de la mort mystérieuse, dans un omnibus allant vers Montmartre, d'une élégante jeune femme, entre le jardin des Plantes et la place Pigalle. Dans la version de Black, Okatsu est effectivement tuée dans un bus traversant Paris de nuit ${ }^{33}$. L'histoire se termine sur l'emprisonnement, malgré son statut social élevé, du businessman Yamada, oncle d'Okatsu, du fait de sa complicité dans le meurtre de sa nièce et de son implication dans la tentative de meurtre de sa sœur Onobu. Or on remarque que la question du règlement approprié de la succession d'Onobu est au cœur de Shachū no dokubari. On se rappelle alors l'importance et la vivacité qu'avaient prises au Japon les débats relatifs à la question des droits des femmes dans le contexte des réformes législatives, et l'on saisit avec quelle intelligence Black sut traiter cette question avec son art. 
comme Amanda Seaman l'a bien remarqué, "l'amalgame entre la narration et la conscience sociale » au Japon permettait aux adeptes de ce genre littéraire d'utiliser de façon intelligente «le narration et les ressources conceptuelles" pour «décrire et critiquer la société japonaise contemporaine $»^{34}$. Le choix de Black d'utiliser le roman policier lui donnait l'opportunité d'illustrer les procédures judiciaires anglaises et françaises et de montrer, à un moment où le droit français constituait une référence importante au Japon, comment les citoyens français étaient tous égaux en droits devant les mêmes lois. Le message que Black adressait donc à ses auditeurs dans son adaptation de l'Épingle empoisonnée de l'omnibus était un message de changement dans la dynamique de domination entre les hommes et les femmes. Le public y découvrait comment la loi française est appliquée à la structure familiale et aux questions de gestion du patrimoine, particulièrement vis-à-vis des droits de succession des femmes.

Dans cette histoire, Black semble d'autre part avoir utilisé le nom de son amant avec qui il habitait alors à Tsukiji, Takamatsu Motokichi, qui devient Kanō Motokichi dans le rôle du Paul Freneuse de la version originale.

\section{Black introducteur de la modernité}

Conformément à la tendance des romans à sensation à donner aux lecteurs des moyens pour mieux s'adapter à la vie moderne, les narrations d'Henry Black contenaient de nombreux récits sur les bienfaits de la science et de la technologie modernes qui permettaient par exemple de résoudre les crimes.

Dans Shachū no dokubari, quand l'étudiant des Beaux-Arts Itō visite la morgue avec la propriétaire du lieu où vivait Okatsu pour identifier le corps de cette dernière, Black en profite pour informer son public sur la relation entre la médecine légale et le droit pénal. Black explique aussi que les cadavres sont employés à des fins médicales si le corps n'est pas réclamé par la famille.

L'étudiant des Beaux-Arts Itō Jirōkichi

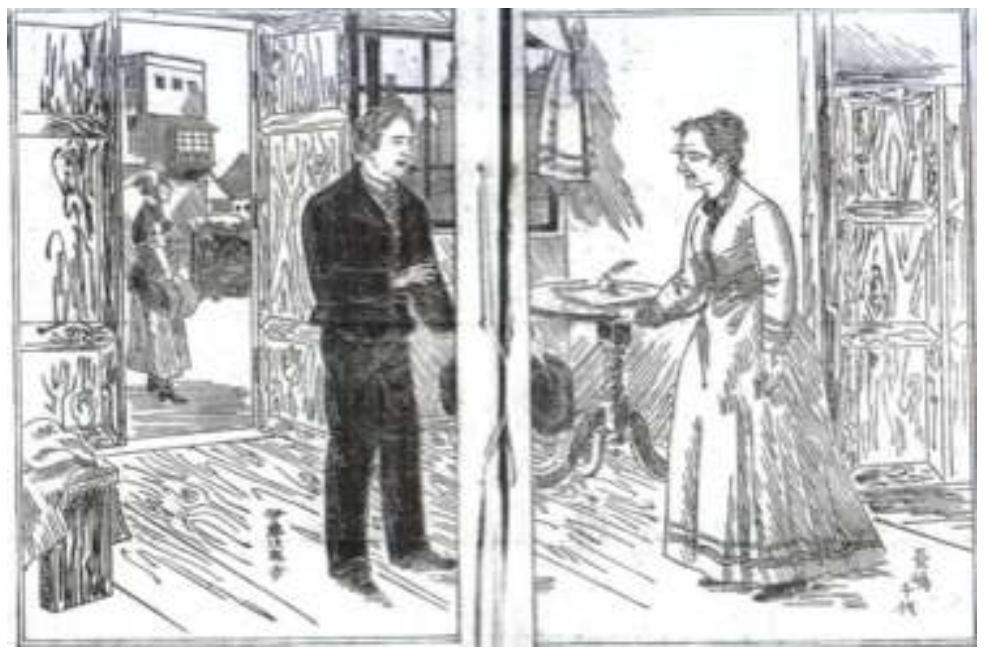

L'étudiant des beaux-arts Itō Jirōkichi tentant de convaincre Nagashima Chiyo, la propriétaire du lieu où vivait Okatsu, de se rendre à la morgue, dans l'Épingle empoisonnée de l'omnibus.

Dans Empreinte de main ensanglantée à la banque Iwade ${ }^{35}$, Black montre comment la police peut très vite traquer Matashichi de Londres jusqu'aux docks de Liverpool, une

Cipango, 20 | 2013 
centaine de ri plus loin (l'équivalent de 393 kilomètres), par le simple biais d'un télégraphe et d'un téléphone permettant de communiquer le profil du suspect à travers tout le pays. Dans cette histoire, le neveu de la victime, Iwade Takejirō, résout l'affaire en comparant une trace de main ensanglantée laissée sur une feuille de papier trouvée sur la scène du crime avec la main d'un employé du service de nettoyage de la banque. L'intrigue, qui s'appuie sur l'usage des empreintes digitales, preuve d'identité considérée comme satisfaisante en Chine et au Japon en l'absence de sceaux gravés, s'inspire peut-être des recherches du docteur Henry Faulds, un missionnaire britannique qui travaillait à l'hôpital de Tsukiji dans les années 1880, soit dans le quartier où Black habitait depuis $1885^{36}$. Le recours par Black aux empreintes digitales en tant qu'élément essentiel du roman policier semble avoir été une première mondiale.

Le thème des réformes législatives comme les descriptions des pratiques judiciaires anglaises et françaises apparaissent souvent dans les histoires de Black. Il en va ainsi de son adaptation du roman de Charles Dickens, Oliver Twist, qu'il intitula L'orphelin ${ }^{37}$. Black y reproduit l'intérêt que Dickens porte à la criminologie, lorsque Nancy se rend au tribunal pour essayer de savoir ce qu'il est advenu d'oliver. Dans la version de Black, la visite incognito d'Omine, l'équivalent japonais de Nancy, lui donne l'occasion d'informer du fait qu'en Angleterre le public est autorisé à assister à la mise en accusation. La scène révèle le soutien enthousiaste de Black aux réformes législatives basées sur les droits civils anglais et français. Ceci apparaît clairement quand on la compare à une scène similaire prise dans l'adaptation déjà évoquée de la nouvelle de Maupassant Un Parricide par son mentor San.yūtei Enchō en 1895.

Le petit Seikichi (Oliver), à gauche, au tribunal devant le juge.

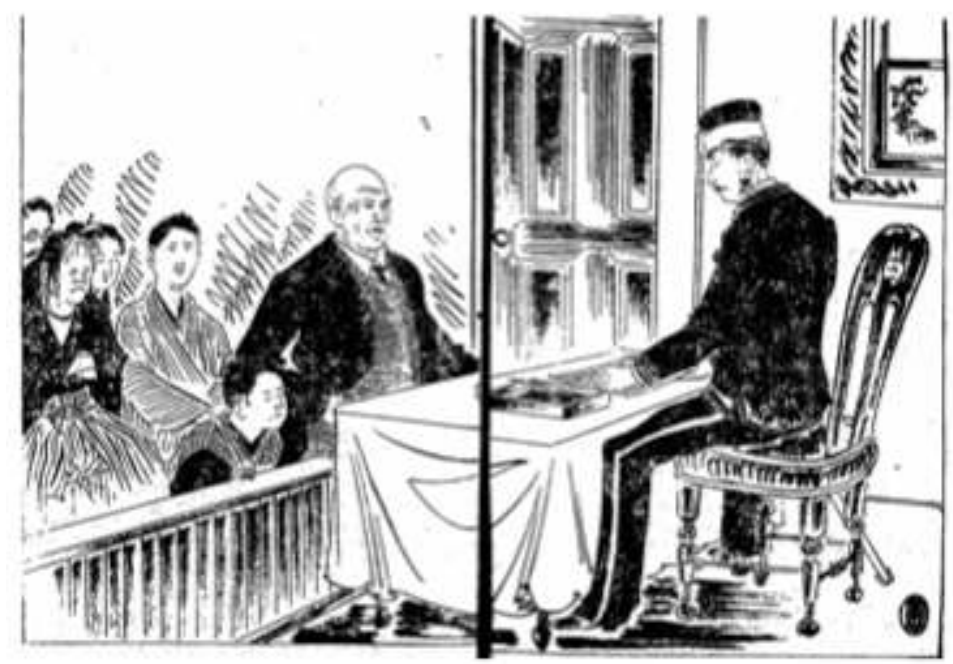

Illustration dans l'adaptation du roman de Charles Dickens, Oliver Twist, que Black intitula Minashigo 孤 児 ('Orphelin).

L'histoire débute au moment où l'artisan est au sommet de son art et de sa gloire. Enchō et Maupassant racontent tous deux la rencontre de celui-ci avec un couple apparemment marié qui se prend d'amitié pour lui et devient son mécène. Chōji suspecte que l'homme et la femme sont en fait ses parents qui l'ont abandonné à sa naissance, et les supplie de lui dire la vérité, mais le couple nie l'évidence. Chōji les tue 
dans un excès de colère. Alors que la version de Maupassant se termine abruptement, laissant aux lecteurs le choix de former leur propre jugement, Enchō consacre lui un temps considérable à décrire le procès et le verdict du juge. Il situe son histoire dans la période Edo, contrairement à Black qui préfère un cadre contemporain, ce qui confère à l'ensemble une teneur nostalgique. Enchō avait de la sorte pour but de plaire à ceux qui voulaient que la morale japonaise classique soit prise en compte dans l'établissement des nouvelles lois. La scène où Chōji se présente devant le juge est radicalement différente de celle racontée par Black dans l'Orphelin. Alors que Black met l'accent sur le droit de tout citoyen à avoir accès à la justice et sur l'égalité de tous devant la loi, Enchō, lui, décrit dans les moindres détails le dialogue entre les protagonistes et met ainsi en relief la différence de statut entre l'humble accusé Chōji et le juge, qui est aussi bienveillant que condescendant. Les illustrations des livrets confirment ces différences. Dans l'orphelin, le voyou Seikichi et le juge sont assis face à face. Seikichi a même ses mains placées sur la barre et ses yeux sont fixés sur le juge. Par contraste, dans les illustrations du Maître ébéniste Chōji, l'accusé est agenouillé, ses yeux sont détournés et sa tête baissée alors que le juge est assis, en dessus, sur une estrade confortable recouverte de tatamis.

Entremêlant avec habileté humour et suspense, les nouvelles d'Henry donnaient les moyens à ses auditeurs et à ses lecteurs de mieux traverser une époque de réformes et d'évolutions rapides de la société.

\section{Black et les nouveaux médias}

Les histoires d'Henry gagnèrent aussi un vaste public au travers des livres et des journaux qui les publiaient de façon fidèle grâce aux notes prises par des sténographes. Cette nouvelle forme de l'oralité jetée par écrit donna naissance à un type particulier de livres appelé sokkibon 速記本 (littéralement « livres [conçus à partir de notes] sténographiques »). L'arrivée au Japon de la sténographie, en 1884, bouleversa le monde de la publication. Le rakugo contribua largement à ce bouleversement. La sténographie fut utilisée au début pour prendre note des débats dans le Parlement tout nouvellement constitué, mais les éditeurs de livres et de journaux adaptèrent très vite cette technique à la publication en masse des récits proposés par les rakugoka populaires. Le coût de ces livres sténographiques, peu élevé grâce à l'emploi de papier de moindre qualité, leur distribution, qui s'appuyait sur un système de bibliothèques de prêt, permirent d'atteindre les masses ${ }^{38}$.

Le premier de ces livres sténographiques reprenait une histoire de fantômes célèbre, la Lanterne pivoine, racontée par San.yūtei Enchō ${ }^{39}$. Ce livre fut si populaire que cinq autres sokkibon des histoires d'Enchō furent publiés l'année suivante.

Si peu de sokkibon ont survécu du fait de la piètre qualité du papier utilisé et parce qu'on ne considérait pas qu'ils méritaient d'être conservés, ces livres se vendirent bien. Bien qu'ils ne fissent pas figure de grande littérature, beaucoup de romanciers et d'éditeurs envièrent leur succès. Leur attrait tenait aussi du fait qu'ils étaient écrits dans un langage simple. En fait, les sokkibon ont servi de "littérature de base pour les populations à peine lettrées, et ont donc aidé les efforts de l'État envers l'alphabétisation $»^{40}$. Ils ont $\mathrm{pu}$ "largement contribuer au renouvellement de la littérature et à la “ révolution " que fut l'avènement du style unifié » (genbun itchi 言文 
一致), en d'autres termes, au mouvement qui servit à «combler le fossé existant jusqu'alors entre les langues écrite et orale $»^{41}$.

À peu près au même moment où le sokkibon se développa, les propriétaires de journaux commencèrent eux aussi à porter un intérêt particulier à la publication sous forme de feuilleton des histoires des rakugoka. Ils devinrent, dans la deuxième partie des années 1880 , un autre moyen pour les conteurs de toucher les masses. Bon nombre des feuilletons publiés étaient des traductions adaptées (hon.an mono 翻案物) d'œuvres françaises ou anglaises, les traducteurs-interprètes donnant des noms japonais aux divers lieux et personnages. Dans certaines des histoires de Black, par exemple, les personnages traversent des ponts à Paris qui portent les noms de ponts bien connus de Tōkyō. Les personnages arrivent dans leur maison à Londres ou à Paris et lisent le Yomiuri shinbun, boivent de la bière et discutent du type de mochi, des gâteaux de riz japonais, qu'ils mangeront pour le Nouvel An. Tout cela crée une mise en scène hybride, permettant ainsi aux lecteurs de s'identifier plus facilement avec les personnages. Le Yamato shinbun やまと新聞 fut un pionnier de cette pratique avec la publication, dès sa première édition, des épisodes de la nouvelle d'Enchō la Belle du pin enterrée vivante (Matsu no misao bijin no ikiume 松の操美人の生埋), en 1886.

Black bénéficia bien sûr de cette diversification des moyens de publication à travers lesquels les conteurs atteignirent un large public. Il avait déjà noué des relations avec les propriétaires de journaux (et en particulier avec le Yamato shinbun), relations qu'il avait développées lorsqu'il travaillait avec son père, éditorialiste, et durant sa collaboration avec le mouvement pour la démocratie, dont on sait les liens qu'il entretint avec la presse de Meiji. Entre 1891 et 1896, le Yamato shinbun, par exemple, publia plusieurs histoires d'Henry, dont Nagare no akatsuki 流扎の暁 (Aube de la rivière ; 45 épisodes entre le début du mois de janvier et la fin du mois de mars 1891), histoire romantique, mais tragique mettant en scène un aristocrate français, le baron Sawanabe, qui fuit la France durant la révolution ${ }^{42}$, mais aussi Setsunaru tsumi 切なる罪 (Péché pitoyable ; 8 mai-21 juin 1891), Tsurugi no hawatari 剣の刃渡り (la Lame de l'épée ; 4 décembre 1892-25 janvier 1893), et Natsu no mushi 夏の虫 (les Insectes de l'été ; 20 décembre 1893-13 janvier 1894). L'éditeur San.yūsha publia par ailleurs, en 1891, la Fille aux roses ${ }^{43}$, roman policier se déroulant à Paris dont le sujet est un complot d'assassinat contre le prince héritier français ${ }^{44}$.

\section{Un artiste protéiforme}

41 On sait que les conteurs visitaient souvent les théatres de kabuki pour observer les expressions faciales et la gestuelle des acteurs, qu'ils incluaient ensuite dans leurs performances. Il était commun qu'ils jouent dans certaines scènes de pièces de kabuki, afin d'asseoir leur notoriété. Henry Black interpréta lui aussi un certain nombre de rôles au kabuji, son préféré étant celui d'un héros du peuple d'Edo, Banzui.in Chōbee 幡 随院長兵衛, qu'il joua pour la première fois au théâtre Haruki 春木座 en septembre 1892. Pour ce rôle, Henry reçut des leçons du fameux acteur Ichikawa Danjūrō, neuvième du nom 九代目市川団十郎 ${ }^{45}$. Danjūrō était un des grands réformateurs du théâtre de l'époque. La coopération entre Danjūrō et Henry était donc une rencontre d'esprits réformistes.

Si le Japan Weekly Mail précisa à cette occasion que Black était sans doute le "premier étranger à monter sur les planches japonaises ", ses interprétations de Banjuin Chōbei 
en 1892 ne constituaient cependant pas sa première incursion dans le monde du kabuki. En 1890, il avait déjà joué des femmes telles qu'Omiwa dans Imoseyama onna teikin 妹背山女庭訓 (Imoseyama, la noblesse d'une femme ${ }^{46}$ ), ou Osato dans Yoshitsune Senbon zakura義経千本桜 (Yoshitsune aux mille cerisiers) ${ }^{46}$, deux pièces qui mettaient en scène le thème de l'amour brisé et une situation où il $\mathrm{y}$ avait erreur sur la personne. En 1891, il avait aussi joué Kumagai, guerrier célèbre, dans le Dit des Heike (Heike Monogatari 平家物語) et Roshishin, moine-brigand dans le récit chinois Au bord de l'eau (Suikoden 水滸伝) ${ }^{47}$. Ces représentations démontrent une immense facilité pour apprendre des dialogues en japonais et les exprimer à la manière d'un Japonais. Son enthousiasme pour le théâtre et son niveau d'admiration pour le Japon étaient sans conteste bien supérieurs à ceux des autres étrangers résidents au Japon.

Henry Black, habillé pour jouer un samouraï (Banzui.in Chōbee)

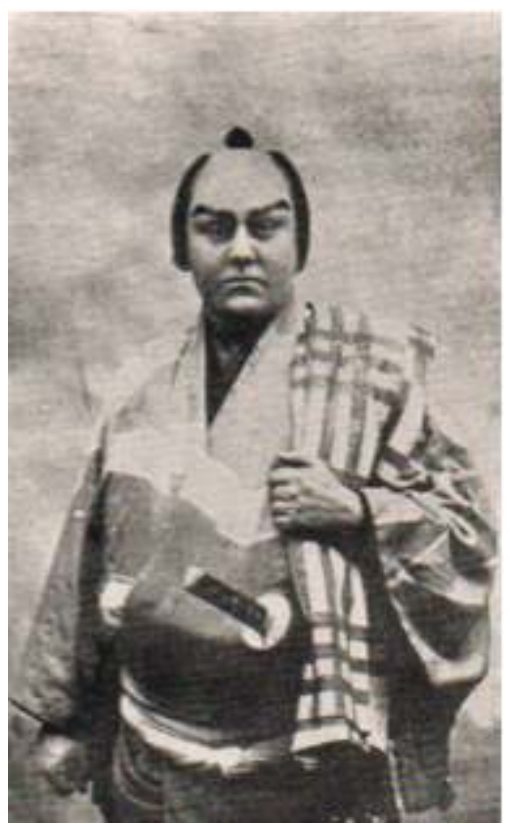

Henry Black, habillé pour jouer un samouraï (Banzui.in Chōbee) ; "The Story Teller in Japan”, par Francis McCullough, in East of Asia Magazine

Henry Black fut également l'un des premiers à enregistrer sa voix sur les tous premiers disques fabriqués au Japon. En 1903, il reçut pour ce faire une aide financière bienvenue de Fred W. Gaisberg, impresario et producteur de disques originaire des États-Unis qui fut le premier à fabriquer des albums pour gramophone en Europe où il travailla pour The Gramophone Company qui détenait le label His Master's Voice ${ }^{48}$. Parmi les disques qu'il produisit figurent les enregistrements d'un groupe composé de "petites femmes geisha accompagnées d'instruments européens ", ce que Gaisberg décrivit lui-même comme étant " la chose la plus amusante qui soit », ainsi que de l'Imperial Household Band et des narrations de Henry Black ${ }^{49}$.

\section{Conclusion}

Henry Black était un homme extraordinaire, un homme qui aimait la vie, le Japon, et le théâtre. Il finit sa vie dans un drôle de ménage avec lequel on retrouve encore la France. Henry adopta en effet un enfant japonais nommé Seikichi, qui épousa plus tard 
une certaine Julie Pequignot. Julie était une personne d'un grand talent. Fille d'un couple français emmenée au Japon par son oncle après la mort de ses parents ${ }^{50}$, elle apprit le shamisen (banjo à trois cordes) et devint une interprète accomplie du récitatif chanté (le gidayū), utilisant comme nom de scène le prénom de Rosa. C'est avec Seikichi et Rosa que Black passa ses dernières années, format un foyer improbable: un Britannique né en Australie et devenu Japonais, son fils adoptif japonais et sa femme d'origine française, Rosa, à Tōkyō.

Henry Black s'éteignit deux semaines seulement après le séisme gigantesque du Kantō, le premier septembre 1923. Le docteur qui signa l'acte de décès attribua la mort à une " sénilité avancée ». Sans consulter ses proches, sa sœur Pauline fit enterrer son corps dans le caveau familial, situé dans le cimetière des étrangers, à Yokohama ${ }^{51}$. Un comble pour celui qui était devenu à ce point Japonais !

Sur sa tombe auraient pu figurer ces mots, tirés de ses Souvenirs du théâtre de Londres :

Si vous rendez visite aux peuples blancs d'Europe, contrairement aux noirs, certains sont tellement blancs qu'ils ressemblent presque à des fantômes sous un clair de lune. Mais leurs sentiments et affections sont les mêmes que ceux des Japonais. Bien que la langue, la tenue vestimentaire, la couleur des yeux ou des cheveux peuvent être différentes, tout être humain possède les mêmes sentiments et émotions ${ }^{52}$.

\section{BIBLIOGRAPHIE}

ADAM Jules, Japanese Storytellers-from the French of Jules Adam, traduit par Osman Edwards, Tōkyō, T. Hasegawa, 1900 (édition française 1899).

ASAOKA Kunio 浅岡邦雄, «Kairakutei Burakku no kekkon/kika mondai kō »快楽亭ブラックの結 婚 - 帰化問題考 (Réflexions sur la question du mariage et de la naturalisation de Kairakutei Black), Kairakutei Burakku Kenkyū 快楽亭ブラック研究, nº 6, 1992, p. 11-12.

BRADDON Mary Elizabeth, Flower and Weed, London, Maxwell, 1884.

BRADDON Mary Elizabeth, "Her Last Appearance", in Weavers and Weft and Other Tales, London, John and Robert Maxwell, 1876.

EIJIN BURAKKU 英人ブラック, Shachū no dokubari 車中の毒針 (l'Epingle empoisonnée de l'omnibus), Tōkyō, Suzuki Kinho 鈴木金補, 1892.

EIKOKUJIN BURAKKU 英国人ブラック,Eikoku Rondon gekijō miyage 英国龍動劇場土産 (Souvenirs du théâtre de Londres), Tōkyō, Nomura Ginjirō 野村銀次郎, 1891.

BURAKKU Henrii ブラック・ヘンリイ Kusaba no tsuyu 草葉の露 (la Rosée du cimetière), Tōkyō, Nisshūsha 日就社, 1886 .

BURAKKU Henrii ブラック・ヘンリイ, Iwade ginkō chishio no tegata 岩出銀行血汐の手形 (Empreinte de main ensanglantée à la banque Iwade), Tōkyō, Azuma Nishiki 東錦, 1893.

FAULDS Henry, "On the Skin-Furrows of the Hand", Nature, n 22-28 October 1880, p. 605.

GAISBERG Fred W., Music on Record, London, Robert Hale Limited, 1946. 
HAMILTON Constance, et YOUNG Michael, "Historical Introduction to the Japanese Legal System", in Curtis J. Milhaupt et al (ed), Japanese Law in Context-Readings in Society, the Economy, and Politics, Harvard University Asia Center, Cambridge, Harvard University Press, 2001.

HANE Mikiso, "The Movement for Liberty and Popular Rights", in Hillary Wray and H. Conroy (eds.), Japan Examined: Perspectives on Modern Japanese History, Honolulu, University of Hawaii Press, 1983, pp. 90-97.

INUMARU Giichi 犬丸義一 (éd.), Shokkō jijō 職工事情 (Conditions des ouvriers d'usine), vol. 1-3, Tōkyō, Iwanami Shoten, 1993.

ISHII BURAKKU 石井ブラック,Minashigo 孤览 (l'Orphelin), Tōkyō, Kin.ōdō 金櫻堂, 1897.

ITō Hideo 伊藤秀雄, Meiji no Tantei Shōsetsu 明治の探偵小説 (Romans policiers de l'ère Meiji), Tōkyō, Shōbunsha 昭文社, 1986.

Japan Weekly Mail, Collection Harold S. Williams, National Library of Australia.

KAIRAKUTEI BURAKKU 快楽亭ブラック, Sobaya no hanashi 蔏麦屋の噺 (Conte du restaurant de soba), EMI Zenshū Nihon fukikomi kotohajime, EMI 全集日本吹き込み事始 (Collection complète EMI sur les débuts des disques vinyles japonais), TOCF-59061-71, nº 8 .

KATSURA Beichō 桂米朝, Rakugo to watashi 落語と私 (le Rakugo et moi), Tōkyō, Popura sha ポプラ 社, 1976.

The Kobe Chronicle, Collection Harold S. Williams, National Library of Australia.

KURATA Yoshihiro 倉田喜弘 (éd.), Meiji no engei 明治の演芸 (les Divertissements de l'ère Meiji), Kokuritsu gekijō chōsa yōseibu geinō chōsashitsu 国立劇場調査養成部芸能調査室 (Centre de recherche sur le divertissement, département de Recherche et d'Éducation, Théâtre national), 1983.

MCARTHUR Ian D., Kairakutei Burakku: Wasurerareta Nippon saikō no gaijin tarento 快楽亭ブラック : 忘れられた日本最高の外人タレント (À la recherche de Kairakutei Black), Kōdansha, Tōkyō, 1992.

MCARTHUR Ian D., “Australian, British, or Japanese? Henry Black in Japan” (Australien, britannique, ou japonais? Henry Black au Japon), Japanese Studies, vol. 22, no 3, 2002, pp. 307-318. MCARTHUR Ian D., Mediating Modernity-Henry Black and Narrated Hybridity in Meiji Japan, Doctoral thesis, University of Sydney, January 2002. http://ses.library.usyd.edu.au/bitstream/ 2123/518/1/adt-NU20030602.10342102whole.pdf

MCARTHUR Ian D., "Henry Black, Rakugo and the Coming of Modernity in Meiji Japan”, Japan Forum, vol. 16, no. 1, 2004, pp. 135-164.

MCARTHUR Ian D., “Why Sonoe Rebuffed Baron Tokiwa: How Mary Braddon Penny Dreadful Reached the Kabuki Stage" (Pourquoi Sonoe repoussa le baron Tokiwa : Comment la romancière à sensation Mary Braddon fut adaptée à la scène Kabuki), The International Journal of Diversity in Organisations, Communities and Nations, vol. 7, no. 2, 2005, pp. 1-14.

MCARTHUR Ian D., “Henry Black: Consummate Meiji Man” (Henry Black : l'homme Meiji accompli), in Michael Ackland and Pam Oliver (eds.), Unexpected Encounters-Neglected Histories Behind the Australia-Japan Relationship (Rencontres inattendues - Les histoires oubliées des relations australo-japonaises), Clayton, Vic., Monash Asia Institute, 2006, pp. 1-28.

MCARTHUR Ian D., "Narrating the Law in Japan: Rakugo in the Meiji Law Reform Debate” (Narrer la législation au Japon : le rakugo dans les débats sur les réformes législatives à Meiji), Electronic 
Journal of Contemporary Japanese Studies, 2008, http://www.japanesestudies.org.uk/articles/2008/ McArthur.html

MCARTHUR Ian D. and Mio BRYCE, "Names and Perspectives in sute obune: A Meiji-Era Adaptive Translation of the Mary Braddon Mystery Novel Diavola" (Noms et perspectives dans sute obune : traduction adaptative du roman mystère de Mary Braddon, Diavola), The International Journal of the Humanities, vol. 5, no. 3, 2007, pp. 141-152.

MCCULLAGH Francis, “The Story-Teller in Japan”, The East of Asia Magazine, vol. 1, Shanghai, North China Herald Office, 1902.

Meiji 26, Naigaikokujin kekkon bo 明治 26 内外国人結婚簿 (Liste de mariages entre Japonais et étrangers), Tōkyō Metropolitan Archives, 604.D5.06 D160 (1-24).

MILLER J. Scott, “Tale as Text: Sokkibon in the Diet Library Collection”, in Helen Hardacre (ed.) with Adam L. Kern, New Directions in the Study of Meiji Japan, Leiden, New York, Köln, Brill, 1997. MOORE Jerrold Northrop, A Matter of Records, Taplinger Publishing Company, New York, 1977. MORIOKA Heinz and SASAKI Miyoko, Rakugo: The Popular Narrative Art of Japan, Cambridge (Massachusetts) and London, The Council on East Asian Studies, Harvard University, 1990. MORIOKA Heinz et SASAKI Miyoko, “The Blue-Eyed Storyteller: Henry Black and his Rakugo Career", Monumenta Nipponica, 38: 2, 1984.

NAKAMURA Kyoko Motomochi (trad.), Miraculous Stories from the Japanese Buddhist Tradition: the Nihon Ryōik of the Monk Kyōkai, Cambridge, Mass., Harvard University Press, 1973.

NISHIYAMA Matsunosuke, "Popular Performing Arts: From Edo to Meiji”, in Matsunosuke Nishiyama, Edo Culture: Daily Life and Diversions in Urban Japan, 1600-1868, Hawaii, University of Hawaii Press, 1997.

NISHŪBASHI Sei 二洲橋生, « Kairakutei Burakku » 快楽亭ブラック,Bungei kurabu 文藝俱楽部, tome 11, vol. 14, Tōkyō, 1905.

NOBUHIRo Shinji 信広真治, Rakugo wa ika ni shite keisei sareta ka 落語はいかにして形成されたか (Comment le rakugo fut formé), Tōkyō, Heibonsha 平凡社, 1986.

NYORAI An 如来庵, «Shunpū Kanwa : Kairakutei Burakku» 春風閑話快楽亭ブラック (Ragots portés par le vent du printemps : Kairakutei Black), Yomiuri Shinbun, 30 avril-3 mai 1896.

OIZURU Yoshiya 生出恵哉, «Chōya shinbun : kōkoku ran ni miru Burakku » 朝野新聞一広告欄に 見るブラック, Kairakutei Burakku kenkyū 快楽亭ブラック研究, nº 1, 19 sept. 1986.

SAKAI Anne, la Parole comme art : le rakugo japonais, Paris, L'Harmattan, 1992.

SAKAI Cécile, Histoire de la littérature populaire japonaise, L'Harmattan, Paris, 1987 (trad. Asahina Kōji 朝比奈弘治, Nihon no taishū Bungaku 日本の大衆文学, 1997).

SAN.YŪTEI Enchō 三遊亭円朝, Meiji bungaku zenshū 明治文学全集 (Collection de littérature Meiji), vol. 10, Tōkyō, Chikuma shobō 筑摩書房, 1965.

SEAMAN A. C., Bodies of Evidence-Women, Society, and Detective Fiction in 1990s Japan, Honolulu, University of Hawaii Press, 2004.

SHIMIZU Yasuyuki 清水康行, «Rekōdo ni kiku Kairakutei Burakku »レコードに聴く快楽亭ブ

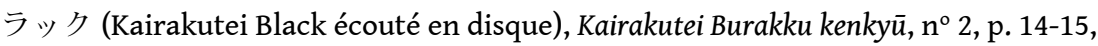
19 septembre 1987. 
SHOGEI Konwakai 諸芸懇話会 (ed.), Kairakutei Burakku kankei bunken mokuroku 快楽亭ブラック文 献目録 (Catalogue de documents concernant Kairakutei Black), Yokohama (sans date).

Yamato shinbun やまと新聞, 24 mars 1891, in Kurata Yoshihiro 倉田喜弘 (ed.), Meiji no engei 明治 の演芸 (les Arts performatifs de Meiji), Kokuritsu gekijō chōsa yōseibu geinō chōsashitsu 国立劇 場調査養成部芸能調查室 (Centre de recherches sur le divertissement, département de Recherche et d'Éducation, Théâtre national), vol. 5, 1983.

YoKOHAMA SHIYAKUSHO 横浜市役所 (Mairie de Yokohama), « Tomitake jidai »富竹亭時代 (l’Ère des Tomitake), in Yokohama shi shikō füzoku hen Dai 5 sō, dai 2 setsu, yose 2 横浜市史稿風俗編第 5 草第 二説寄席 2 (Annales de la ville de Yokohama, t. 5, vol. 2, yose 2), Yokohama, 1973.

YoSHIZAWA Hideaki 吉沢英明, Taishū geinō shiryō shūsei 大衆芸能資料集成 (Collection de documents sur les divertissements populaires), vol. 5, Tōkyō, San.ichi shobō, 1981.

ZWICKER Jonathan E., Practices of the Sentimental Imagination, Harvard University Asia Center, Cambridge, Mass., Harvard University Press, 2006.

\section{NOTES}

1. D'excellentes discussions sur les origines, l'histoire et les caractéristiques du rakugo dans Heinz MORIOKA et Miyoko Sasaki, Rakugo: The Popular Narrative Art of Japan, Cambridge (Massachusetts) and London, The Council on East Asian Studies, Harvard University, 1990 ; Anne SAKAI, La Parole comme art - le rakugo japonais, Paris, Éditions l'Harmattan, 1992.

2. Ou Nihon reiiki 日本霊異記, dont une traduction anglaise est disponible: Kyoko Motomochi Nakamura, Miraculous Stories from the Japanese Buddhist Tradition: the Nihon Ryōiki of the Monk Kyōkai, Cambridge, Mass., Harvard University Press, 1973.

3. MORIOKA et SASAKI, op. cit., p. 239-241; SAKAI, op. cit., p. 67-76.

4. MORIOKA et SASAKI, op. cit., p. 310-321.

5. MORIOKA et SASAKI, op. cit., fournissent une liste complète de titres.

6. SAKAI, op. cit., p. 81-98.

7. J. Scott MILLER, "Tale as Text: Sokkibon in the Diet Library Collection", in Helen HARDACRE (ed.), with Adam L. KERN, New Directions in the Study of Meiji Japan, Leiden, New York, Köln, Brill, 1997, p. 582.

8. Francis mccullagh, "The Story-Teller in Japan", The East of Asia Magazine, vol. 1, Shanghai, North China Herald Office, 1902, p. 207.

9. KATSURA Beichō 桂米朝, Rakugo to watashi 落語と私 (le Rakugo et moi), Tōkyō, Popurasha ポプ ラ社, 1976, p. 86.

10. NOBUHIRO Shinji 延広真治, Rakugo wa ikani shite keisei saretaka 落語はいかにして形成された か (Comment le rakugo prit forme), Tōkyō, Heibonsha 平凡社, 1986, p. 20; NISHIYAMA Matsunosuke, "Popular Performing Arts: From Edo to Meiji", in Edo Culture: Daily Life and Diversions in Urban Japan, 1600-1868, Hawaii, University of Hawaii Press, 1997, p. 247.

11. SAN.YŪTEI Enchō, Meiji bungaku zenshū 明治文学全集 (Collection de littérature Meiji), vol. 10, Chikuma shobō, 1965, p. 336-375.

12. MORIOKA et SASAKI, op. cit., p. 257 ; Anne SAKAI, op. cit., p. 83-93.

13. Pour de plus amples informations sur les annonces de journaux donnant la liste de ces meetings et leurs sujets, voir oIzURU Yoshiya 生出恵哉, “Chōya shinbun : kōkoku ran ni miru Burakku»「朝野新聞」広告欄にみるブラック (Chōya shinbun : Black au travers des colonnes publicitaires), dans Kairakutei kenkyū 快楽亭研究, nº 1, 19 septembre 1986, p. 10-11. 
14. L'intérêt des Japonais pour Napoléon découla en particulier de leur curiosité à l'égard de l'expérimentation européenne de diverses formes de gouvernement et codes pénaux, ainsi que du désir du gouvernement japonais de surimposer un style parisien à l'aménagement des grands boulevards de Tōkyō.

15. «Tomitake jidai » 富竹時代, in Yokohama shi shikō füzoku hen 横浜市史稿風俗編 (Annales de la ville de Yokohama - Les pratiques urbaines), tome 5, vol 2, Yose 寄席 2, Yokohama Shiyakusho 横浜市役所, 1973, p. 252-254.

16. EIKOKUJIN Burakku 英国人ブラック, Kusaba no tsuyu 草葉の露 (La rosée du cimetière), Tōkyō, Nisshūsha 日就社, 1886.

17. Mary Elizabeth BRADDON, Flower and Weed, Maxwell, London, 1884. Mary Braddon fait partie de ces nombreux auteurs à sensation de l'ère victorienne, qu'ils soient d'origine britannique, française ou américaine, qui furent traduits et popularisés au Japon durant la décennie précédant l'accession de Black au statut de shin.uchi.

18. MORIOKA and SASAKI, dans "The Blue-Eyed Storyteller", op. cit., p. 141, citent une annonce parue dans l'édition du 17 septembre 1890 de l'Asahi shinbun.

19. NISHŪBASHI Sei 二洲橋生, «Kairakutei Burakku », in Bungei kurabu 文藝俱楽部, 1905, tome 11, vol. 14, Tōkyō, p. 298. Black avait confié à son intervieweur : « Seiyōjin no rakugoka wa mezurashii to iu no de, tachimachi koi ga ōhyōban ni narimashita » 西洋人の落語家は珍しいというので、たちま ちこいが大評判になりました ( un rakugoka occidental étant considéré peu ordinaire, je devins rapidement populaire »).

20. «Rakugoka chū ni isshu keiro no kawatta tokoro o motte uridashita eijin burakku. » 落語家中に一種 毛色の変わった処を以て売出した英人ブラック, cité in KURATA Yoshihiro 倉田善弘 (éd.), Meiji no engei 明治の喜芸 (les Spectacles de l'ère Meiji), Tōkyō, Kokuritsu gekijō chōsa yōseibu geinō chōsa shitsu 国立劇場調査養成部芸能調査屋, vol. 5, p. 97.

21. Jules ADAM, Japanese Storytellers-from the French of Jules Adam (Édition française 1899), traduction Osman Edwards, Tōkyō, T. Hasegawa, 1900, ici p. 19 de l'édition anglaise, retraduit en français par l'auteur.

22. Ibid., p. 21-22.

23. L'adoption du gendre par mariage n'est pas une pratique rare en effet.

24. Chūō shinbun, 24 mai 1893, cité in KURATA, op. cit., p. 177-178.

25. Naigaikokujin kekkon bo 内外国人結婚簿 (Liste des mariages entre Japonais et étrangers), Meiji 26 (1893), Tōkyō Metropolitan Archives, 604.D5.06 D160, p. 1-24.

26. «Hijōni Takamatsu Motosuke narumono o ai shi hotondo füfu mo tadanaranu arisama非常に高松元 助ナルモノヨ愛し殆ど夫婦も旁ならぬ有様》; document de neuf lignes intitulé Keihi 京秘 $\mathrm{n}^{\circ} 286$, signé par le superintendant en chef de la police de Kyōbashi, le 28 avril 1893; Naigaikokujin kekkon bo, op. cit., p. 14-15. La note de police fait mention de « Motosuke» 元助, mais d'autres documents, y compris un livre co-publié avec Henry, attestent l'usage du nom Motokichi 元吉. La question du mariage de Black a déjà été évoquée par Asaoka Kunio 浅岡邦雄, «Kairakutei Burakku no “Kekkon/Kika” Mondai kō»快楽亭ブラックの「結婚・帰化」問題考 (Réflexions sur la question du mariage et de la naturalisation de Kairakutei Black), Kairakutei Burakku Kenkyū, nº 6, 1992, p. 11-12.

27. KAIRAKUTEI Burakku, Sobaya no hanashi 䒾麦屋の噺 (Conte du restaurant de soba), EMI Zenshū Nihon fukikomi kotohajime EMI 全集日本吹き込み事始 (Collection complète EMI des débuts des vinyls japonais), TOCF-59061-71, no 8.

28. The Kōbe Weekly Chronicle, no. 234, vol. 9, 24 December 1901, p. 623. L'article attribuait ce commentaire au Japan Herald. Extrait dans Harold S. Williams Collection, National Library of Australia.

29. Mary Elizabeth BRADDON, "Her Last Appearance", dans Weavers and Weft and Other Tales, London, John and Robert Maxwell, 1876. 
30. EIKOKUJIN Burakku 英国人ブラック,Eikoku Rondon gekijō miyage 英国倫敦劇場土産 (Souvenirs du théâtre de Londres, en Angleterre), Nomura Ginjirō 野村銀次郎, Tōkyō, 1891.

31. Braddon a en effet souvent été accusée d'avoir plagié les travaux d'autrui, y compris ceux d'auteurs populaires contemporains anglais et français. On la sait avoir été friande des œuvres de Zola, Flaubert et Balzac. Certains éléments suggèrent que même si elle ne plagiait pas directement ces auteurs, elle a été grandement influencée par eux.

32. EIJIN Burakku 英人ブラック, Shachū no dokubari 車中の毒針 (L’Épingle empoisonnée de l’omnibus), Suzuki Kinho 鈴木金補, Tōkyō, 1892.

33. Dans les adaptations de Black, les noms de lieux demeurent généralement fidèles à l'original, sauf lorsqu'un effet humoristique est recherché. Par contre, les noms de personnes sont souvent remplacés par des noms japonais.

34. Amanda C. SEAMAN, Bodies of Evidence-Women, Society, and Detective Fiction in 1990s Japan, Honolulu, University of Hawaii Press, 2004, p. 1.

35. Henry BLACK, Iwade ginkō chishio no tegata 岩出銀行血汐の手形 (Empreinte de main ensanglantée à la banque Iwade), Azuma Nishiki 東錦, Tōkyō, 1893.

36. Dans un article intitulé "Au sujet des rides de la main », publié dans le journal scientifique britannique Nature, Faulds expliqua qu'il avait eu l'idée de se lancer dans ses recherches après avoir appris que les potiers japonais laissaient leurs empreintes digitales sur l'argile de leurs pots pour permettre d'identifier leur provenance ; Henry FAULDS, "On the Skin-Furrows of the Hand", Nature, $22-28$ oct. 1880, p. 605.

37. ISHII Burakku 石井ブラック，Minashigo 抓児 (l'Orphelin), Kin.ōdō 金桜堂, Tōkyō, 1897. On constate que Black publie désormais sous son nom japonais, acquis par le mariage.

38. Concernant l'introduction de la sténographie et son application au sokkibon, voir J. Scott MILLER, op. cit., p. 581-589; Jonathan E. ZWICKER, Practices of the Sentimental Imagination, Harvard University Asia Center, Cambridge, Mass., Harvard University Press, 2006, pp. 66-67 et 90-92.

39. SAN.YŪTEI Enchō, Botan dōrō 牡丹燈籠, in Meiji bungaku zenshū, vol. 10, Chikuma shobō, 1965, p. 3-82.

40. Miller, op. cit., p. 583.

41. Anne Sakai à propos de la langue d'Enchō, op. cit., p. 90-91.

42. MORIOKA et SASAKI, op. cit., p. 142. L'histoire contient des éléments qui rappellent le professeur de français Charles Darnay dans le roman de Dickens A Tale of Two Cities, qui est centré sur les personnages de deux vrais frères jumeaux, l'un bon, l'autre dissolu.

43. Bara musume 著薇娘; ibid., p. 144. Il semblerait que l'histoire intitulée Kokuji Tantei 国事探偵 (L'inspecteur public) et publiée par Kikuike Yūhō 菊池幽芳 dans l'ōsaka mainichi shinbun 大阪毎 日新聞 en 1898 proviendrait de la même source, peut être d'origine européenne ; Itō Hideo 伊藤 秀雄, Meiji no tantei shōsetsu 明治の探偵小説 (Les romans policiers de Meiji), Futabasha 双葉社, p. $165-188$.

44. L'anarchiste Ohana projette de se déguiser en fleuriste pour attirer le prince dans un piège et l'assassiner à l'aide d'un gaz mortel. Lorsque le chef de la police de Paris, Kanamachi Shō, apprend que le scientifique et opposant politique Nishino Takeshi est entré sur le territoire avec l'intention d'assassiner le prince héritier, il charge l'inspecteur Ōmura de l'enquête.

45. Japan Weekly Mail, 3 September 1892, p. 275.

46. Cette œuvre a été traduite en français sous le titre Imoseyama ou l'éducation d'une femme par Jeanne SIGÉE, Gallimard, 2009.

46. MORIOKA et SASAKI, op. cit., p. 142 , citent des comptes rendus de ces représentations apparaissant dans l'édition du 17 septembre 1890 de l'Asahi shinbun.

47. Asahi shinbun, 24 mars 1891. SHOGEI Konwakai (éd.), Kairakutei Burakku kankei bunken mokuroku 快楽亭,ブラック関係文献目録, p. 4 .

48. Fred W. GAISBERG, Music on Record, London, Robert Hale Limited, 1946, p. 59. 
49. Jerrold Northrop mooRe, A Matter of Records, New York, Taplinger Publishing Company, 1977, p. 82.

50. Entretien personnel avec un des petit-fils de Seikichi, M. Sudō Mitsuo, à Shizuoka, le 3 mai 1991; Heinz MORIOKA et Miyoko SASAKI, Kairakutei Burakku no 'Nippon' 快楽亭ブラックの ニッポン (Le Japon de Kairakutei Black), Tōkyō, PHP Kenkyūjo PHP研究所, 1986, p. 183-184.

51. Ces détails sur les circonstances de la mort de Black me proviennent d'une interview privée avec la fille de Rosa, Mme Ishii Kiyoko, le 3 mai 1991.

52. Eikoku Rondon gekijō miyage, op. cit., p. 41.

\section{RÉSUMÉS}

Dans les années 1880-1890, l'utilisation de la sténographie pour publier de la façon la plus fidèle les récits des conteurs professionnels connus sous le nom de rakugoka permit à ces derniers d'être connus et appréciés d'un très large public. C'est au cours de cette période qu'un citoyen britannique d'origine australienne, Henry Black (1858-1923), se fit conteur professionnel et raconta des histoires se déroulant en Grande-Bretagne ou en France afin de faire comprendre à un public populaire les changements sociaux initiés par le gouvernement de Meiji. Ses histoires constituaient des modèles pour aider les Japonais à entrer dans la modernité de la fin du XIx siècle. Elles illustraient les réformes juridiques, les nouvelles relations entre les hommes et les femmes et la nouvelle urbanité. Black fut en ce sens un participant enthousiaste des débats de l'ère Meiji sur le sens de la modernité.

In the 1880s-1890s, the use of stenography for the publication of narrations by professional storytellers known as rakugoka enabled their stories to be printed and read by mass audiences. During this period, Australian-born British citizen, Henry Black (1858-1923), adapted sensation fiction from Britain and France to illustrate for mass audiences social change instigated by the Meiji government's reform agenda. His stories were templates for assisting Japanese people to negotiate nineteenth-century modernity. The stories contained examples of legal code reforms, new male-female relationships and new notions of the city. Black was an enthusiastic participant in the Meiji debate over the meaning of modernity.

\section{INDEX}

Mots-clés : conte, rakugo, Black Henry James (1858-1923), culture populaire

Thèmes : arts du spectacle

Keywords : rakugo, Tale, Black Henry James (1858-1923), Popular Culture, Meiji Period, Performing Arts

Index chronologique : Meiji (1868-1912) 
AUTEUR

IAN MCARTHUR

Université de Sydney 